\title{
Large Scale Multiphase Flow Numerical Simulation of Individualized Thoracic Aortic Dissection
}

\author{
Zhang Jinxin ${ }^{1,}$, Zhang Hongming ${ }^{\text {1, b, * }}$, Zhang Linxiang ${ }^{1}$, and Zhang Jinhu ${ }^{2}$ \\ ${ }^{1}$ Department of Engineering Mechanics, Kunming University of Science and Technology, kunming 650500, \\ P.R.China \\ ${ }^{2}$ Yanan Hospital, kunming kunming 650500,P.R.China \\ a1354403213@qq.com, bhmzhang8888@126.com \\ *Corresponding author
}

Keywords: aortic dissection, multiphase flow, numerical simulation, particles

\begin{abstract}
Objective To further explain the blood flow characteristics of artery dissection by numerical simulation. Methods The CT (Computed Tomography) scan results of artery dissection were transformed into three-dimensional flow field in CFD(Computational Fluid Dynamics) software to build Lagrange multiphase flow simulation containing red blood and plasma by MIMICS (Materialise's interactive medical image control system). Results Vortex appears in the proximal end of false lumen, the inlet of false lumen and it's opposite experience relatively strong wall share stress, pressure differ between the two sides of the membrane, and is bigger in the distal end of false lumen, The distribution of red blood cells is uneven. Conclusions Arterial dissection reduces the amount of red blood cells in the blood; due to the wall share stress and pressure, proximal end of false lumen damage easily; holes appear on the membrane beside branch arteries and balance pressure and flow; the arterial dissection is prone to thrombosis.
\end{abstract}

\section{Introduction}

Aortic dissection (AD) is a disease that the blood of the aorta enters the outer layer or the junction of the middle and outer layer of the aorta media. It can initiate rupture of the aorta which may cause death of the patient, and it led to obstruction of the true lumen which may cause the important organs (intestines, kidneys, lower limbs, etc.) have an ischemic change and cause a serious complication $^{[1,2]}$.

Hemodynamic analysis of aortic disease is an important progress of computational fluid dynamics (CFD) in the field of biomedicine in recent years ${ }^{[3-10]}$. Some studies have used analyzed the aorta through geometric modeling ${ }^{[3,4]}$. However, other studies have shown that small differences in the geometric shape of the cardiovascular system may greatly affect the distribution of shear stress on the wall of the vessel [8], which means that the method of geometric modeling is not accurate enough. Some studies use the generalized non-Newtonian fluid to simulate the blood ${ }^{[3-7]}$, while others use multiphase to do that ${ }^{[9,10]}$. Compared the two method, obviously the multiphase flow simulation can better reflect the real situation of the blood, and can get important data (distribution, trajectory, flow etc.) of red blood. However, multiphase flow simulation often using Euler method, which regard the red blood cell as liquids mixed in the blood, instead of regarding that as particles for calculation.

\section{Research method}

\section{1 geometric model}

Firstly, this article constructs a geometric model based on a patient's CT scan. Then, analysis of the characteristics of blood mechanics and erythrocyte movement by the method of Lagrange 
multiphase flow. By this way, providing scientific basis for the treatment and understanding of aortic dissection, as well as, providing more reference cases.

According to the CT image of the patient's chest, the three-dimensional model of the STL format is constructed by using the medical software MIMICS, and importing the model into CFD software. After that, divide and identify the surface of the model and then divide elements. It's remarkable that the fluid region is divided by polyhedron mesh, and the mesh's base size is $0.1 \mathrm{~mm}$. The boundary of the whole fluid region is divided by 5 layers of prism layer mesh, and 5 million 500 thousand units are obtained.

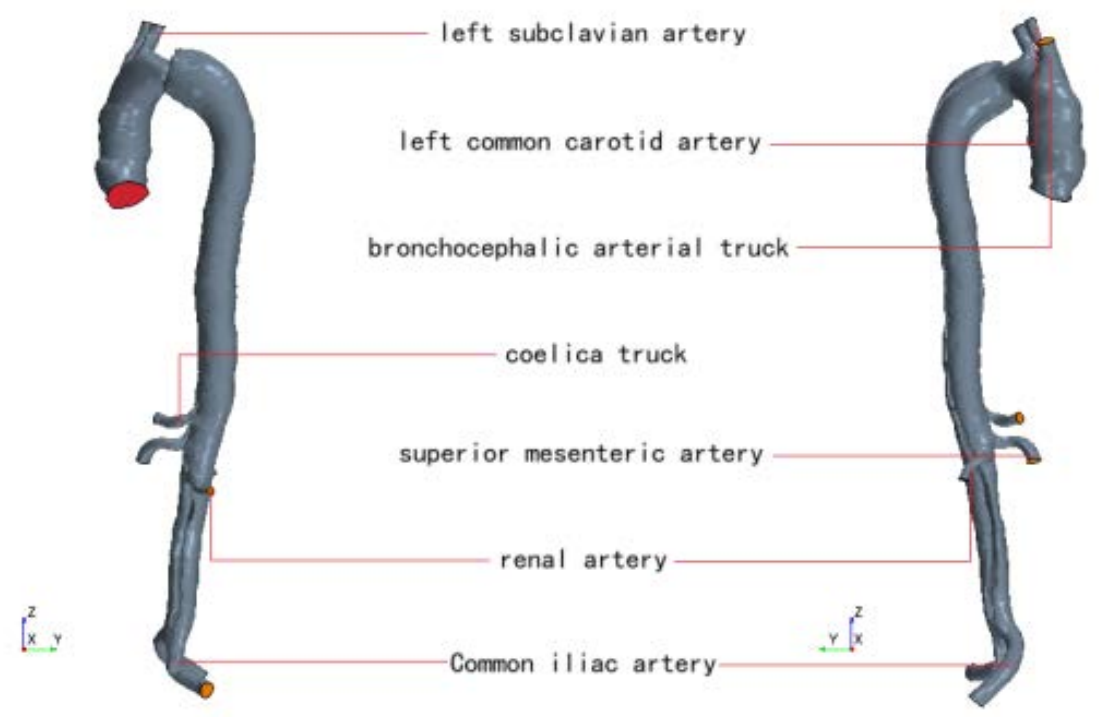

Fig.1 Three dimensional dissection of aortic dissection.

\section{2 numerical method}

In this simulation, Lagrange multiphase flow is used for calculation. The plasma is defined as a continuous phase, and the red cell is defined as a granular phase, which are mixed by volume ratio with $45: 55^{[10]}$. The blood flow velocity of a patient who have artery dissection was measured at the entrance speed ${ }^{[2]}$. The whole calculation repeating ten cycles.

The continuous phase using the unsteady incompressible fluid and the Navier-Stokes equation, as well as the finite volume method. And the dynamic viscosity is $0.0035 \mathrm{~Pa} \cdot \mathrm{s}^{[10]}$. The diffusion term using center difference method. The advection Scheme using second-order upwind scheme. The time term using implicit scheme.

The particle phase is calculated by Lagrange multiphase flow ${ }^{[11]}$, and the simulation is set up based on the correlation equation of the CFD software Lagrange multiphase flow. Basic motion equation:

$$
\frac{d r_{p}}{d t}=v_{p}-v_{g}
$$

$v_{g}(x, t)$--grid speed; $v_{p}(t)$-- absolute speed of particles; $r_{p}(t)$-- the position of the particle with respect to the frame of reference; The particle terms are given the subscript $\mathrm{p}$.

Descartes coordinates are used in this simulation. Variable $t$ discrete in time, and the time step is $0.005 \mathrm{~s}$.

The equation of conservation of mass of a material particle is:

$$
\frac{d m_{p}}{d t}=\dot{m}_{p}
$$

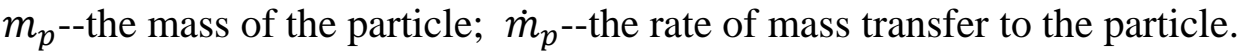

In this paper, the particle simulation of red blood cells, which does not involve chemical changes and changes of state, so $\dot{m}_{p}=0$.

The generic form of the equation of conservation of momentum for a material particle is: 


$$
m_{p} \frac{d v_{p}}{d t}=F_{S}+F_{b}
$$

$F_{S}$--the forces acting on the surface of the particle; $F_{b^{-}}$-the body forces.

These forces in turn are decomposed into:

$$
F_{b}=F_{g}=F_{d}+F_{p}+F_{v m}
$$

$F_{d^{-}}$-the drag force; $F_{p^{-}}$-the pressure gradient force; $F_{v m}$--the virtual mass force; $F_{g^{-}}$-the gravity force.

The equation for the drag force is:

$$
F_{d}=\frac{1}{2} C_{d} \rho A_{p}\left|V_{s}\right| V_{s}
$$

$C_{d^{--}}$the drag coefficient of the particle, value in the text is $0.0175 \mathrm{~Pa} \cdot \mathrm{s}^{[10]}$; $\rho$--the density of the continuous phase, value in the text is $1090 \mathrm{~kg} \cdot \mathrm{m}^{-3}{ }^{[10]}, V_{s}$--the particle slip velocity; $A_{p^{--}}$-the projected area of the particle. The particle radius value in the text is $8 \mu \mathrm{m}{ }^{[10]}$.

The equation for the pressure gradient force is:

$$
F_{p}=-V_{p} \nabla P_{\text {static }}
$$

$V_{p^{-}}$-the volume of the particle; $P_{\text {static }}$--the gradient of the static pressure in the continuous phase.

The equation for the virtual mass force is:

$$
F_{v m}=C_{v m} \rho V_{p}\left(\frac{D V}{D_{t}}-\frac{d V_{p}}{d t}\right)
$$

$C_{v m}$--the virtual mass coefficient, the default value of 0.5 for this coefficient is for a sphere in a uniform, inviscid, incompressible flow. The operator D/Dt is the material (substantive) derivative.

The equation for the gravity force is:

$$
F_{g}=m_{p} g
$$

$g$-- the gravitational acceleration vector, value is $9.8 \mathrm{~m} \cdot \mathrm{s}^{-2}$, according to the position of the import model, the direction is in the negative direction of the $\mathrm{Z}$ axis.

The equation for the energy balance is:

$$
m_{p} c_{p} \frac{d T_{p}}{d t}=Q_{t}+Q_{\text {rad }}+Q_{S}
$$

$Q_{t}$--the rate of convective heat transfer to the particle from the continuous phase; $Q_{\text {rad }}{ }^{\text {--the rate }}$ of radiative heat transfer; $Q_{S}$--other heat sources. There is no thermal change in this simulation, and the equation is 0 on both sides of the equation.

\section{Result}

Using 128 cores to parallel compute for 65.7 hours, this calculation simulates the blood flow of aortic dissection within ten heartbeat cycles. In order to reduce the influence of the initial conditions, tenth periodic data is analyzed.

\subsection{Velocity field analysis}

The blood velocity varies with the speed of the entrance, and the position of $7.7 \mathrm{~s}$ reaches the maximum. The blood flow velocity of the broncho cephalic arterial truck, the left common carotid artery, and the left subclavian artery is the largest in several branches of the artery. The farther the entrance to the entrance, the slower the arterial branch changed. The flow of blood in the aorta is mainly laminar flow, and many whirlpools occur near the entrance of the false cavity and the re breach of the abdominal artery, which is in accordance with the records of the Bluth and other ${ }^{[14]}$. The high speed blood flows through narrow entrance of the false cavity, and is bound by the blood 
vessel to form whirlpools. The velocity is gradually reduced to the end of the heart. The velocity of the false cavity near the end of the heart is very low. In the position of the abdominal aorta, the re breaks often appear near the branch outlet, and they balance the speed on both sides.
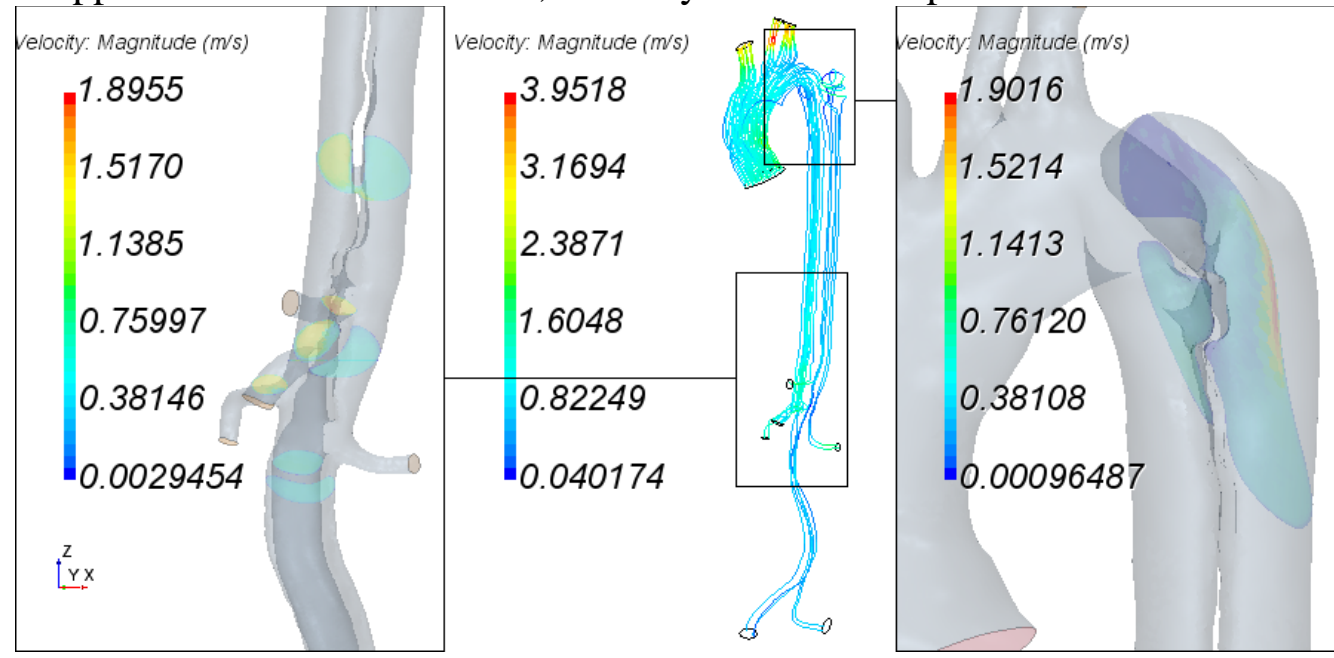

Fig.2 T=7.7s streamline and special sections velocity scalar diagram.

\subsection{Wall stress analysis}

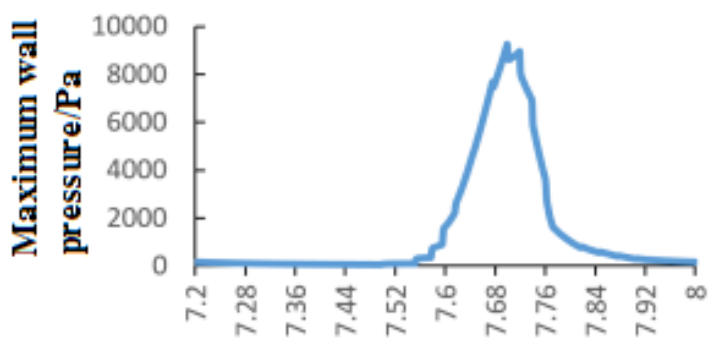

time/s

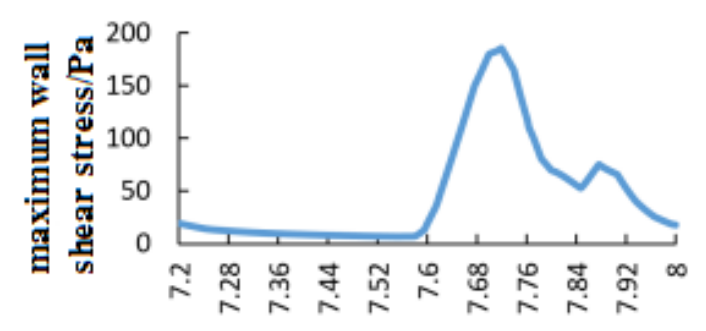

time/s

Fig.3 Maximum wall pressure and maximum shear stress diagram.
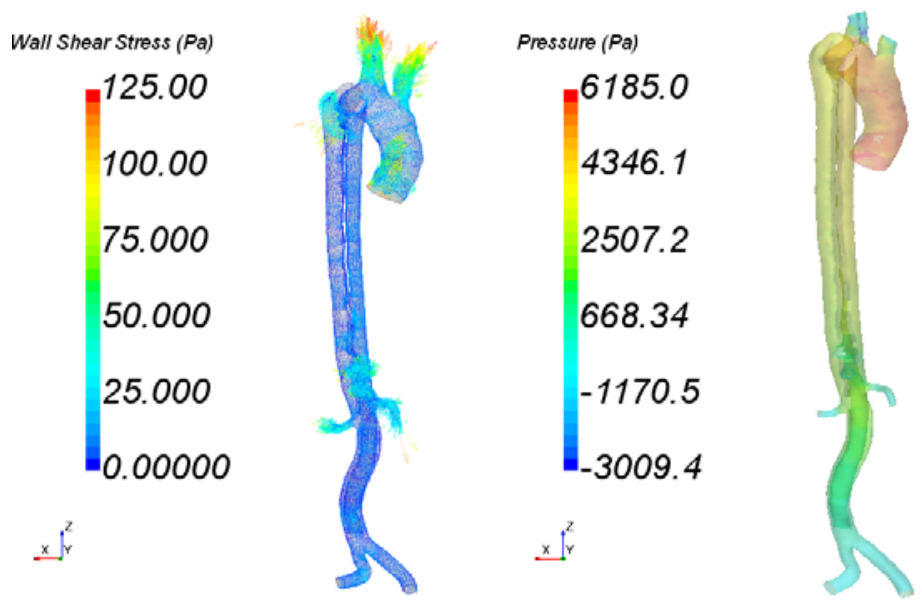

Fig.4 T=7.68s Wall shear stress and Wall pressure diagram.

The maximum shear stress and maximum pressure of the wall fluctuate with the inlet velocity, and the maximum shear stress and pressure in the cycle occur near the 7.68s. There is a large wall shear stress on the entrance of the false lumen and its contralateral vessel wall, the re breaks, and the initial branch of the branch artery. The other locations are basically homogeneous, and the values of wall shear stress are small. The wall pressure of the ascending aorta is significantly larger. The pressure in the true cavity is generally higher than that of the false cavity, and the pressure of 
the true cavity falls quickly to the distal end of the heart, and the declines of the false cavity are slow.

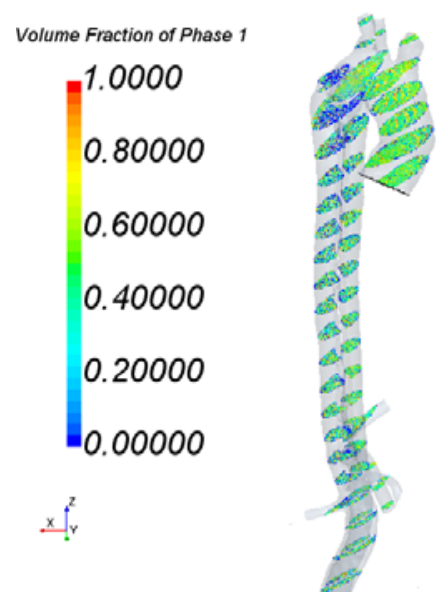

Fig.5 T=7.72s erythrocyte volume fraction diagram.

\subsection{Analysis of red blood cell distribution}

During the tenth cycles, the entrance rate of 7.58s to 7.86s is large, and a large number of red cells enter the calculation area. This stage is the main time for the transport of red blood cells and the volume fraction of red blood cells in the computational domain is obviously improved. The distribution of red blood cell volume fraction is similar, and the image of 7.72s is analyzed as the representative. The volume of red blood cell in ascending aorta was high, the volume of red cell in the proximal part of the false lumen was very low, the volume of red blood cell in the abdominal artery was homogeneous, the volume fraction of red blood cells near the re breaks was slightly lower, and the volume of the true chamber red blood cell was higher than that in the false lumen.

\section{Discussion}

In the tenth period, the speed of the entrance is maximum at 7.7s. Figure 2 shows that the wall pressure and shear stress also almost reach the maximum at this time. The periodic change of entry speed has a major influence on the force of the arterial wall. This indicates that patients can control heart rate by proper way of life to reduce the deterioration of arterial dissection. However, the red blood cell volume fraction does not fully satisfy this rule. Therefore, the movement of red blood cells can not be completely judged by the entry velocity and the continuous phase velocity. It is necessary to simulate the blood as Lagrange multiphase flow. Red blood cell volume fraction decreased from ascending aorta to descending aorta, and the volume of red blood cells in arterial dissection was relatively low, which reflected that arterial dissection has a hindrance to erythrocyte movement. For the same reason, the red blood cell volume fraction at the middle layer of the abdominal artery is low. Previous medical experience has suggested that arterial dissection reduces the blood transfusion capacity of the aorta. Not only that, arterial dissection can reduce the content of red blood cells in blood by blocking red blood cells.

In figures 3 and 5, due to the narrowing of the tear, the speed of the blood flowing into the false lumen is very high, resulting in a larger shear stress near the tear, which makes the tear fracture continue to be damaged, inflamed and expanded. The change of wall pressure is more uniform. The pressure of the wall of the ascending aorta, the wall of the false cavity and the wall of the true cavity have wall pressure decreased in turn. The wall of the proximal end of the cavity of the false cavity is subjected to a relatively large shear stress, which is easily damaged under the condition that it has been stretched and thinned. This result is caused by the high speed blood flowing into the false chamber, and forming vortex of which speed reaches $1.9 \mathrm{~m} \cdot \mathrm{s}^{-1}$ near the wall. By observing the angle of the tear and combining the dilatation of the false cavity, the doctor can make a rough discrimination of the vulnerable position. 
In Figure 3, it is found that the pressure and velocity of the true cavity and the false cavity are very different, and the pressure and flow velocity near the re breaks are similar. The re breaks help to balance the flow velocity on both sides, relieve the pressure of the false cavity and reduce the extrusion of the false cavity to the true cavity.

The majority of the blood flowing into a false cavity flows to the distal end of the heart, and a few flows to the proximal end of the heart to form a low-speed vortex. Low speed whirlpool makes red blood cells, fibrin and other substances in the blood gather and stay, and form thrombus. The expanded proximal end of the false cavity suffering the shear stress is easy to be inflamed, making it easier to form a thrombus here. Thrombus may adhere to the wall of the vessel, clog the blood vessels and cause rupture.

\section{Conclusion}

(1) The change of the entrance velocity has a very obvious effect on the wall share stress and pressure of arterial dissection. It is of great significance to avoid severe exercise to prevent the deterioration of the disease.

(2) Artery dissection not only reduces the blood transfusion ability of the aorta, but also hinders the passing of red blood cells to reduce the content of red blood cell particles in the blood, leading to low blood oxygen carrying capacity.

(3) The proximal end of the false cavity is affected by pressure and wall shear stress. The effect of shear stress is more obvious, and it produces damage and even rupture.

(4) Re breaks help balance the pressure and flow rate of both sides;

(5) At the proximal end of the false cavity, it is easy to form a thrombus and clog the blood vessel.

\section{References}

[1] Wang Liang. Computational Fluid Dynamics Numerical simulation analysis of Stanford B type aortic dissection[D], Shanghai, The Second Military Medical University, 2010.

[2] Wang Liang, Lu Qing-sheng, etc. Preliminary study on the hemodynamic numerical simulation of Debakey III type aortic dissection[J]. Journal of Interventional Radiology,2010,19(9),683-687.

[3] Wang Wen-quan,Yan Yan, Zhang Cheng-lei . Numerical Simulation of Blood Pulsatile Flow in a DeBakey III Type of TVD[J]. Journal of Beijing University of Technology.2012,38(6),949-954.

[4] Zhang Yage, Gao Bin, Chang Yu. The effect of left ventricular assist device (LVAD) cannula anastomosis location on micro-emboli movement in aorta[J]. Journal of Medical Biomechanics,2016,31(3):199-205

[5] Chu Bo, Qiao Ai-ke. Fluid-structure interaction numerical simulation of bypassed DeBakey III aortic dissection. Journal of Medical Biomechanics[J].2012,27(4).386-391.

[6] Wang Peng, Zhang Xi-wen, Fan Zhan-ming. Numerical calculation of abdominal aortic dissection based on computed tomography scan[J]. Engineering Mechanics.2012,29(1).229-234.

[7] Li Bai, Li Xiao-yang. The influence from microcirculation load based on numerical simulation of two-way fluid-structure coupling of aorta[J]. Journal of Medical Biomechanics, 2016,31(5):426-430

[8] Johnston Barbara M, Johnston Peter R, Corney Stuart, et al. Non-Newtonian blood flow in human right coronary arteries: steady state simulations[J]. Journal of Biomechanics, 2004, 37(5): 709-720.

[9] Zeng Yu-jie, Luo Kun, Fan Jian-ren, etc. Aortic Dissection Blood Two-Phase Flow Numerical Simulation Study [J]. Journal of Engineering Thermophysics.2016.37(4).780-784.

[10] Yang Jin-you, Yu Hang, Liu Jing, etc. Numerical Simulation in two-phase Blood Flow in 
Stenosis of Vertebral Artery[J]. Progress in Modern Biomedicine, 2014,14(18):3410-3413.

[11] starccm+ userguild 11.02[Z]:4295-4309.

[12] Milne-Thompson, L.M. Theoretical Hydrodynamics[M]. New York: McMillan \& Co, 1968.

[13] Bluth EI, Murphey SM, Hollier LH, Sullivan MA. Color flow Doppler in the evaluation of aortic aneurysms[J]. INTERATIONAL ANGIOLOGY, 1990;9(1):8 